\title{
Photon structure functions at the ILC energy range
}

\section{Beata Krupa ${ }^{1}$, Bogdan Pawlik, Tomasz Wojtoń and Leszek Zawiejski}

Institute of Nuclear Physics PAN

Cracow, Poland

E-mail: beata.krupa@ifj.edu.pl

On behalf of the FCAL Collaboration

\begin{abstract}
The measurement of photon structure function in the future $\mathrm{e}^{+} \mathrm{e}^{-}$collider ILC energy range will improve our understanding of the QCD. In order to check the possibility of measuring the photon structure functions at this collider the simulations of two-photon processes were performed at the ILC centre-of-mass energy range using Monte Carlo generators and the ILCSoft package. The analysis used the information obtained from the forward detectors, tracking detectors and calorimeters which are parts of the ILD detector concept.
\end{abstract}

The European Physical Society Conference on High Energy Physics

22-29 July 2015

Vienna, Austria

${ }^{1}$ Speaker

(C) Copyright owned by the author(s) under the terms of the Creative Commons

Attribution-NonCommercial-NoDerivatives 4.0 International License (CC BY-NC-ND 4.0). 


\section{Introduction}

The discovery of the Higgs particle at the LHC [1, 2], currently the most powerful accelerator, was an essential step in achieving the present level of our understanding of the fundamental interactions of nature and the structure of matter described by the Standard Model. However, there are still many questions to answer. For instance, the Standard Model does not explain dark matter. Therefore, future experiments are planned and the construction of a new electron-positron collider is much anticipated. Such accelerators, both linear (ILC in Japan [3] and CLIC at CERN [4]) and circular (FCC at CERN [5] and CEPC in China [6]), are currently in the phase of intense studies. Future accelerators will be precise research tools that will allow us to explore the nature beyond the knowledge acquired from the LHC. The research program for future experiments is very rich. One possibility would be to measure the photon structure functions.

The first measurement of the photon structure functions was performed using the PLUTO detector at the DESY storage ring PETRA (1981) [7]. Following this pioneering work, many experiments have been performed at all high energy $\mathrm{e}^{+} \mathrm{e}^{-}$and ep storage rings, where the lepton beams serve as a source of high energy photons. In spite of extensive research, the photon is so far not understood at the same level as HERA has achieved for the proton. This will offer new insights into QCD. Recent experimental data have been derived from the experiments at LEP [8]. New data can be obtained from a new linear $\mathrm{e}^{+} \mathrm{e}^{-}$collider.

\section{Method of measuring the photon structure functions}

The photon as a gauge boson of QED is a massless and chargeless particle with no internal structure in the common sense. However, according to quantum field theory the existence of interactions carried by a gauge boson means that this boson can develop some structure. The photon, for example, can fluctuate for a short period of time into a charged fermion - antifermion pair carrying the same quantum numbers as the photon. It can therefore interact with other particles directly as a whole quantity (direct photon) or through particles produced by the quantum fluctuations (resolved photon) (see e.g. [9]). The diversity of the photons' behaviour allows us to study its leptonic or hadronic structure. The quantities used to describe this structure are the photon structure functions.

The classic way to investigate the photon's structure at $\mathrm{e}^{+} \mathrm{e}^{-}$colliders is the study of the process: $\mathrm{e}^{+} \mathrm{e}^{-} \rightarrow \mathrm{e}^{+} \mathrm{e}^{-} \gamma^{(*)} \gamma^{(*)} \rightarrow \mathrm{e}^{+} \mathrm{e}^{-} \mathrm{X}$, proceeding via the interaction of two photons, which can be either quasi-real (close to the mass-shell) or virtual (off-shell) [10]. The incoming leptons radiate photons producing a hadronic or leptonic final state $\mathrm{X}$. The kinematics of these two-photon interactions is illustrated in Figure 1, which also includes the definitions of the photons' virtualities $\left(\mathrm{Q}^{2}\right.$ and $\mathrm{P}^{2}$, where $\mathrm{P}^{2}$ means the virtuality of the target photon) and the invariant mass squared of the $\gamma \gamma$ system $\left(\mathrm{W}^{2}\right)$. The polar angles $\theta_{\mathrm{i}}, \mathrm{i}=1,2$ at which the electrons are scattered are measured with respect to the direction of beam electrons and depend on the virtualities of the photons.

In the case where only one of the scattered beam electrons can be detected (single-tagged event), the process can be described as deep inelastic electron scattering off a quasi-real photon (er DIS). These events can be studied to measure both the QED and the hadronic photon 


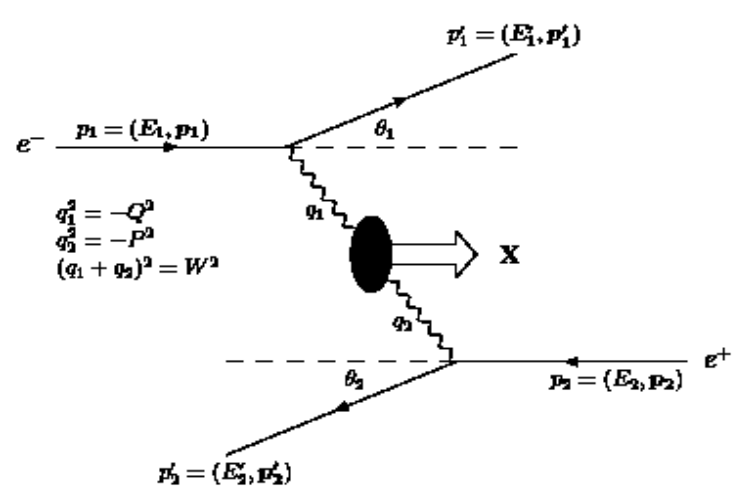

Fig. 1. Kinematics of the two-photon process [8].

structure functions [9], analogously to the studies of the proton structure functions at HERA [11]. The measurements of the QED structure functions are possible by analysing the events with lepton pairs in the final state. Because of the low statistics and difficulty with the identification of the $\tau^{+} \tau^{-}$pairs as well as the number of different Feynman diagrams contributing to the process with $\mathrm{e}^{+} \mathrm{e}^{-}$pairs, the cleanest measurement from the experimental point of view can be performed for $\mu^{+} \mu^{-}$final state. Whereas the contribution to the hadronic photon structure functions comes from processes with hadronic final states.

Experimentally, the kinematical variables used to describe the process of deep inelastic e $\gamma$ scattering: the virtuality $\mathrm{Q}^{2}$, the Bjorken variable $x$ and the inelasticity $y$, are obtained from the four-vectors of the tagged electrons and the hadronic or leptonic final state using the information about the polar angle $\theta$ at which the tagged electron is scattered and their energy E (discussed in detail in [12]) via:

$$
\begin{aligned}
& \mathrm{Q}^{2}=4 \mathrm{E}_{\mathrm{b}} \operatorname{Esin}^{2}\left(\frac{\theta}{2}\right), \\
& \mathrm{x}=\frac{\mathrm{Q}^{2}}{\mathrm{Q}^{2}+\mathrm{W}^{2}+\mathrm{P}^{2}}, \\
& \mathrm{y}=1-\frac{\mathrm{E}}{\mathrm{E}_{\mathrm{b}}} \cos ^{2}\left(\frac{\theta}{2}\right),
\end{aligned}
$$

where $E_{b}$ is the energy of the beam electrons. The differential cross section can be written in terms of $x$ and $y$ as [10]:

$$
\frac{\mathrm{d}^{2} \sigma}{\mathrm{dQ^{2 }} d \mathrm{x}}=\frac{2 \pi \alpha^{2}}{\mathrm{x} \mathrm{Q}^{4}}\left(\left[1+(1-\mathrm{y})^{2}\right] \mathrm{F}_{2}^{\gamma}\left(\mathrm{x}, \mathrm{Q}^{2}\right)-\mathrm{y}^{2} \mathrm{~F}_{\mathrm{L}}^{\gamma}\left(\mathrm{x}, \mathrm{Q}^{2}\right)\right)
$$

where $F_{2}^{\gamma}\left(x, Q^{2}\right)$ and $F_{L}^{\gamma}\left(x, Q^{2}\right)$ are the photon structure functions. In the present experimental conditions $y$ is quite small in general, so it is very difficult to measure $\mathrm{F}_{\mathrm{L}}^{\gamma}$ [8]. Therefore the experiments usually neglect $\mathrm{F}_{\mathrm{L}}^{\gamma}$ and focus on the measurement of $\mathrm{F}_{2}^{\gamma}$, a procedure justified by theoretical considerations based on $\mathrm{QCD}$ that $\mathrm{F}_{2}^{\gamma}$ is the leading component. The structure function $\mathrm{F}_{2}^{\gamma}$, as defined in Eq. (4), is extracted from the differential cross section.

Since the beam energy at the ILC/CLIC will be higher than at the previous experiments, it is expected that it will be possible to measure the evolution of the photon structure functions 
in a wider kinematical range than presently available. Moreover, at the largest values of $\mathrm{Q}^{2}$ one could measure the contributions from $\mathrm{Z}^{0}$ exchange in deep inelastic e $\gamma$ scattering and the charged-current process e $\gamma \rightarrow v \mathrm{X}$, mediated by a virtual $\mathrm{W}$ boson could be used to study the weak structure of the photon [13]. Another very important improvement would be the possibility of detecting the electron which radiates the quasi-real photon. This electron is scattered under almost zero angle. Tagging both electrons would allow $\mathrm{W}^{2}$ to be measured independently of the hadronic final state. Then the precision of the determination of the photon structure functions may be significantly improved $[9,14]$. Additionally, new light on the photon structure could be shed by spin-dependent structure functions (more details in $[15,16]$ ), which have not been measured so far. This would be possible using polarized electron and positron beams in the future linear collider. Furthermore, two-photon processes are a background for physical analyses in which signals from the so-called new physics are being searched for, and their evaluation will be important to obtain reliable results [17].

\section{Results from Monte Carlo simulations}

For the study of the possibility to measure QED and hadronic photon structure functions at the future linear $\mathrm{e}^{+} \mathrm{e}^{-}$collider, the simulations of two-photon processes were performed at the ILC centre-of-mass energy equal to $500 \mathrm{GeV}$ using Monte Carlo generators and the ILCSoft package [18]. The analysis used the information related to the forward detectors, tracking detectors and calorimeters which are parts of the ILD (International Large Detector) detector concept [3]. The results of PYTHIA 6.4 [19] Monte Carlo studies as well as those obtained using the reconstructed kinematic variables in the case of tagging the scattered electrons in deep inelastic e $\gamma$ scattering with the special calorimeters in the very forward region, $\mathrm{LumiCal}^{2}$ and $\mathrm{BeamCal}^{3}$ detectors [20], are presented in Figure 2. These forward detectors allow scattered electron tagging in the regions $31-78 \mathrm{mrad}$ and $5.8-43.5 \mathrm{mrad}$ respectively. There has been observed large detector effects which will require correcting due to detector acceptance (the so-called unfolding method). The uncertainties marked on the plots are statistical only. Of course, the systematic effects should be also estimated. This will be, besides the consideration of possible background processes, the next step of the analysis.

Because the study is based on the Monte Carlo generations of single-tagged events in deep inelastic e $\gamma$ scattering regime it is important to select a Monte Carlo generator, which enables to describe these processes at the energy range of the future experiment. It is thus intended to compare the PYTHIA generator level results with results from other Monte Carlo generators, such as the latest version of WHIZARD [21] and those used at the LEP experiments (HERWIG, TWOGAM) [22].

The initial comparison of the PYTHIA generator level results with the predictions of the HERWIG and TWOGAM generators has been made. Figure 3 shows the centre-of-mass energy $W_{\mu \mu}$ and polar angle distributions of a two muon system obtained using different generators in the case of the QED photon structure's study. Various generators give different values of kinematic variables, therefore further investigation is necessary in order to find out what is the reason of this discrepancy and which results are the closest to the true value.

\footnotetext{
${ }^{2}$ The LumiCal will be the luminometer of the experiment using Bhabha scattering as the gauge process.

${ }^{3}$ The BeamCal will be used to measure remnants of beamstrahlung to assist beam tuning and also high energy single electrons.
} 

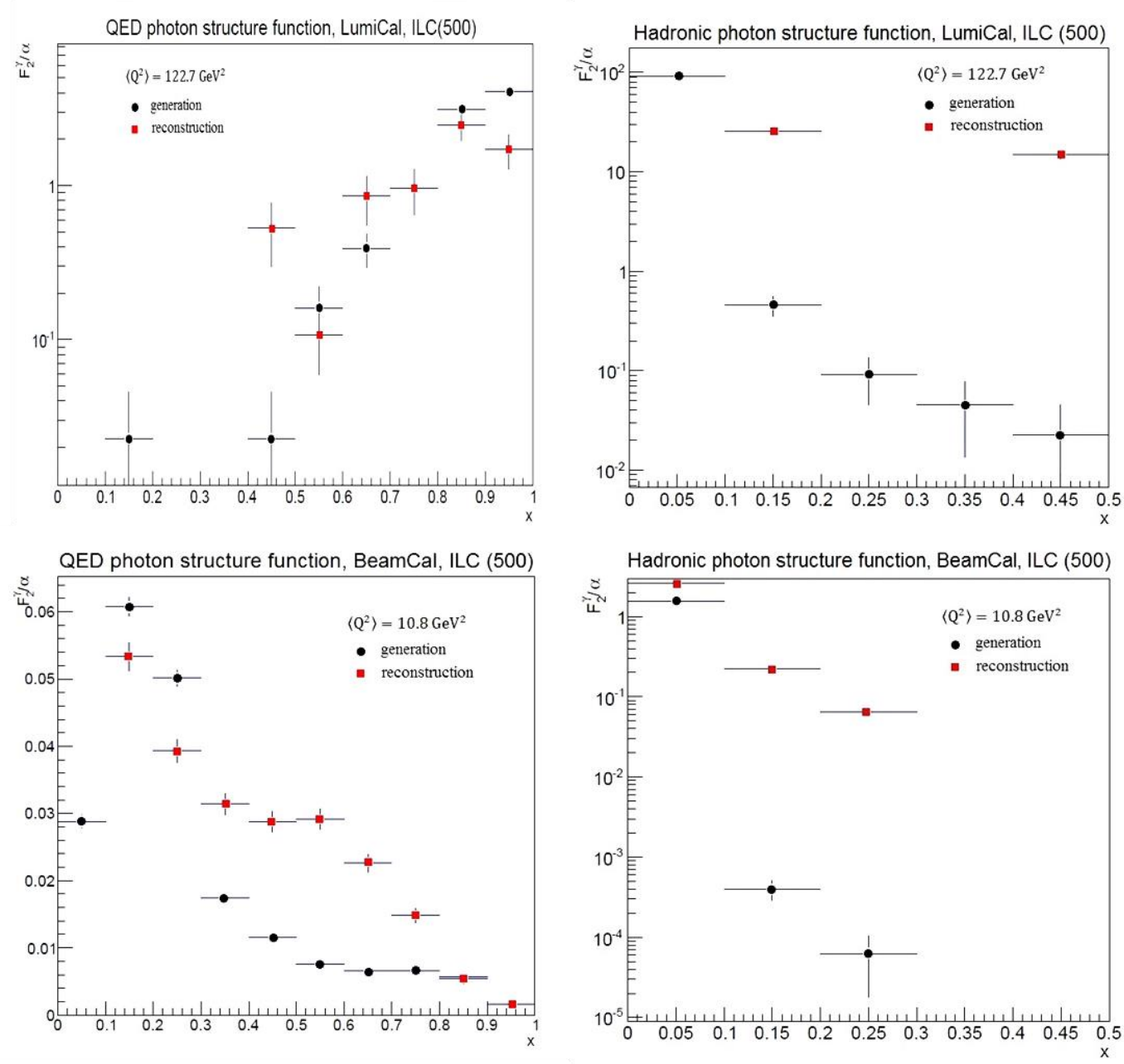

Fig. 2. The QED and hadronic photon structure functions divided by the fine structure constant as a function of $\mathrm{x}$ variable in the case of tagging the scattered electrons at LumiCal and BeamCal detectors (generation and reconstruction levels).
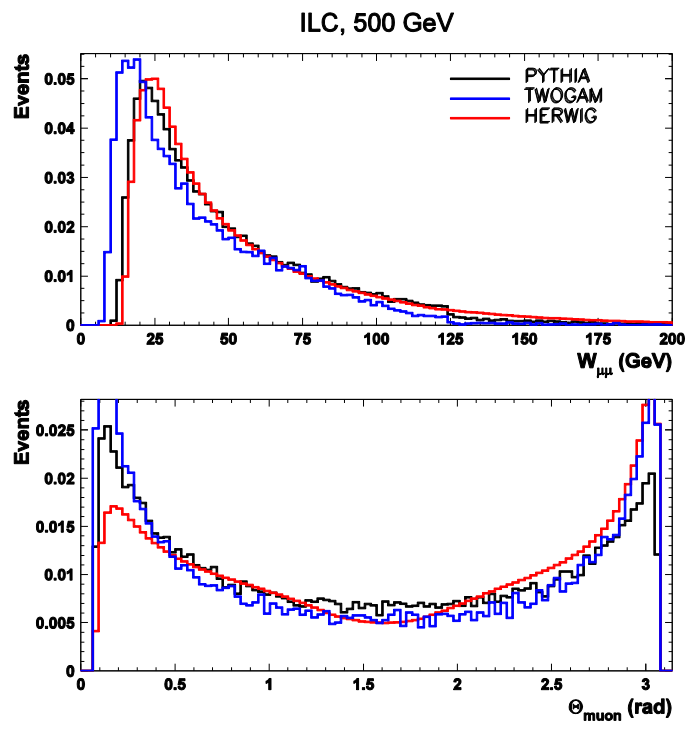

Fig. 3. The centre-of-mass energy $\mathrm{W} \mu \mu$ and polar angle distributions of a two muon system obtained using different generators in the case of the QED photon structure's study. 


\section{Summary}

Further investigations of the photon structure are undoubtedly necessary and they would greatly benefit from the measurements performed at the future linear $\mathrm{e}^{+} \mathrm{e}^{-}$collider. Therefore studies on the potential of the ILC/CLIC in this field of research are needed. In order to check the possibility of measuring the photon structure functions at the future experiment the simulations of two-photon processes were performed using various Monte Carlo generators and the ILCSoft package. The obtained results indicate that the forward detectors, LumiCal and BeamCal, can be used for tagging electrons scattered at e $\gamma$ DIS and would be accordingly useful in the measurement of the photon structure functions.

The next step towards a complete analysis will include the consideration of possible background processes (beamstrahlung, annihilation, etc.) and the estimation of systematic uncertainties. Moreover, the determined kinematical variables should be corrected with regard to detector effects (the so-called unfolding method). It is also intended to compare the results with theoretical predictions at NLO QCD.

\section{References}

[1] G. Aad et al., Observation of a new particle in the search for the Standard Model Higgs boson with the ATLAS detector at the LHC, Phys. Lett. B 716 (2012) 1 - 29.

[2] S. Chatrchyan et al., Observation of a new boson at a mass of $125 \mathrm{GeV}$ with the CMS experiment at the LHC, Phys. Lett. B 716 (2012) $30-61$.

[3] A. Abada et al., The International Linear Collider Technical Design Report, ILCREPORT-2013-040 (ISBN 978-3-935702-74-4), 2013.

[4] P. Lebrun et al., CLIC Conceptual Design Report, CERN-2012-005, 2012.

[5] FCC H2020 Project, H2020-INFRADEV-1-2014-1, 2014.

[6] Y. Wang, Introduction to CEPC - SPPC, talk presented at the Future Circular Collider Study Kickoff Meeting, Geneve, 2014 [indico. cern. ch/event/282344/].

[7] PLUTO Collaboration, Ch. Berger et al., First measurement of the photon structure function $F_{2}$, Phys. Lett. B 107 (1981) 168-172.

[8] L3 Collaboration, P. Achard et al., Measurement of the photon structure function $F_{2}^{\gamma}$ with the L3 detector at LEP, Phys. Lett. B 622 (2005) 249-264.

[9] R. Nisius, The photon structure from deep inelastic electron - photon scattering, Physics Reports 332 (2000) 165-317 [hep-ex/9912049]

[10] R. M. Godbole, Photon structure function, 1996 [hep-ph/9602428]

[11] H1 Collaboration, F. D. Aaron et al., Measurement of the inclusive ep scattering cross section at low $Q^{2}$ and $x$ at HERA, Eur. Phys. J. C 63 (2009) 625-678.

[12] V. M. Budnev et al., The two-photon particle production mechanism. Physical problems. Applications. Equivalent photon approximation, Physics Reports 15 (1975) 181-282.

[13] A. Gehramann-De Ridder, H. Spiesberger, P. M. Zerwas, Deep inelastic ey scattering at high $Q^{2}$, Phys. Lett. B 469 (1999) 259-262. 
[14] R. Nisius, Two photon physics at the future linear collider, 1998 [hep-ex/9811024v1]

[15] M. Glück, E. Reya, C. Sieg, Spin dependent structure functions of the photon, Phys. Lett. B 503 (2001) 285-291.

[16] K. Sasaki, T. Uematsu, Mass effects in the polarized virtual photon structure, Nucl. Phys. Proc. Suppl. 135 (2004) 178-182.

[17] R. Sahara, T. Uematsu, Y. Kitadono, Photon structure function in supersymmetric QCD revisited, Phys. Lett. B 707 (2012) 517-522.

[18] ILCSoft, http://ilcsoft.desy.de/portal/

[19] T. Sjöstrand, S. Mrenna, P. Skands, PYTHIA 6.4 - physics and manual, IHEP 05 (2006) 026.

[20] FCAL Collaboration, H. Abramowicz et al., Performance of fully instrumented detector planes of the forward of a Linear Collider detector, JINST 5 (2010) P12002.

[21] W. Kilian, T. Ohl, J. Reuter, WHIZARD: Simulating Multi-Particle Processes at LHC and ILC, Eur. Phys. J. C 71 (2011) 1742 [hep-ph/0708.4233]

[22] L. Lönnblad, $\gamma \gamma$ event generators, in proceedings of the Workshop on Physics at LEP2, 10.5170/CERN-1996-001-V-2, 187. 